\title{
Transmission, refraction, and focusing properties of labyrinth based left-handed metamaterials
}

\author{
Ekmel Özbay", Irfan Bulu, and Humeyra Caglayan \\ Nanotechnology Research Center, Department of Physics, \\ Department of Electrical and Electronics Engineering, Bilkent, 06800 Ankara, Turkey
}

Received 23 September 2006, revised 26 December 2006, accepted 27 December 2006 Published online 22 March 2007

\section{PACS 77.22.Ch, 78.20.Ci, 81.05.Zx}

In this present article, we studied the transmission, refraction and focusing properties of one, two and three dimensional labyrinth based left-handed metamaterials. We demonstrated that the proper periodic arrangement of labyrinth structures and wires results in left-handed transmission. By using a two dimensional labyrinth based left-handed slab, it is shown that it is possible to image the field emitted from source in sub-wavelength detail. Moreover, we demonstrated the focusing and negative refraction of electromagnetic waves by a three dimensional labyrinth based left-handed slab.

(C) 2007 WILEY-VCH Verlag GmbH \& Co. KGaA, Weinheim

\section{Introduction}

The electromagnetic properties of materials are described by the use of two parameters: permittivity and permeability. The permittivities of known naturally occurring materials can attain positive and negative values. On the other hand, the permeabilities of known naturally occurring materials are known to be positive. However, there is no fundamental reason implied by the laws of electromagnetism that may restrict the values of permittivities and permeabilities to positive values [1]. As a matter of fact, it has been shown that when a medium attains negative permittivity and permeability, simultaneously, it may exhibit certain very exciting properties such as negative refraction [2-4], left-handed transmission, reverse Doppler shift [1,5], and subwavelength focusing [6-9]. While nature does not provide us with a material with simultaneous negative permittivity and permeability, this obstacle has been overcome by the use of metamaterials. By use of the metamaterials, which are artificially created structures, we may now engineer the properties of a medium so that the medium exhibits the desired permittivities and permeabilities.

In this present study, we investigated the transmission, refraction, and focusing properties of one, two, and three dimensional labyrinth based metamaterial mediums. In the next first two sections, we will summarize our results on the one and two dimensional labyrinth based left-handed metamaterials, in which we first showed that the labyrinth based metamaterial medium exhibits a left-handed transmission band over a certain frequency range [10]. Moreover, we showed that the magnetic resonance of the labyrinth structure cannot be excited by electric coupling. In Section 3, we investigated the focusing properties of a two dimensional medium [8]. Our results demonstrate that it is possible to focus the source image to subwavelength dimensions by use of a flat labyrinth based left-handed slab. In Section 4, we presented our recent results regarding the three dimensional case.

\footnotetext{
* Corresponding author: e-mail: ozbay@bilkent.edu.tr, Phone: +90 31229019 66, Fax: +90 3122901015
} 


\section{Transmission properties of one dimensional medium}

Split ring resonator (SRR) is a commonly used structure in order to obtain negative permeability [11-22]. While SRR structure provides negative permeability and can be used to obtain negative refraction, it has several disadvantages. One of these disadvantages is the bianisotropy. Several authors have shown that a medium consisting of a periodic arrangement of SRRs is bianisotropic [23-25]. The bianisotropy is the result of the non-zero electric dipole moment of the SRR structure due to the asymmetric placement of slits on the rings. Second, it has been shown that the magnetic resonance of the SRR structure can be excited via electric fields [26]. The excitation of the magnetic resonance of the SRR structure results from the capacitive coupling of the electric field. The capacitive coupling of the electric field creates non-zero current along the rings. These two disadvantages make it difficult to obtain isotropic, homogeneous two or three dimensional negative refraction media by using SRRs for negative permeability. We should note that these two disadvantages are directly related to one another.

We proposed the labyrinth structure in order to overcome these specific disadvantages of the SRR structure. The labyrinth structure consists of four rings instead of two. The two additional rings are used for two main purposes of which the first is to enhance the strength of the resonance. Second, the two-ring structure has two magnetic resonances that are close to each other. We used the additional two rings in order to separate the two magnetic resonances further away in frequency from each other. The unit cell of the fabricated structure is shown in Fig. 1. The structures are fabricated by using the standard printed circuit board manufacturing methods.

The transmission properties of a single labyrinth structure were characterized by using a HP $8510 \mathrm{C}$ vector network analyzer and two monopole antennas as the receiver and transmitter antennas. The measured and calculated transmission spectrum of plane EM waves through a single layer of a labyrinth structure is shown in Fig. 2(a). We used CST Microwave Studio in our calculations. The directions of the electric field, magnetic field, and wave vector of the incident EM waves are shown in the insets of Fig. 2(a) and (b). First of all, the transmission spectrum of a single labyrinth structure exhibits a resonance around $6.2 \mathrm{GHz}$ with a transmission of $-25 \mathrm{~dB}$ (Fig. 2(a)). Second, the closed labyrinth structure does not exhibit this resonance in the transmission spectrum. As a result, the transmission spectrum shown in Fig. 2(a) clearly demonstrates that the labyrinth structure exhibits a magnetic resonance around 6.2 GHz. Note that for the case of incidence configuration shown in Fig. 2(b) the magnetic resonance of the SRR structure can be excited by electric fields. The measurement and calculation results for a single

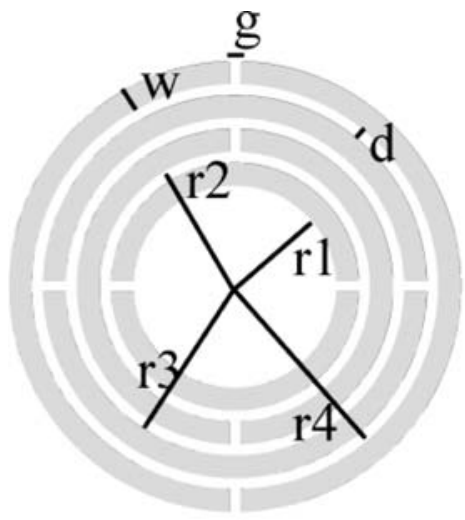

a)

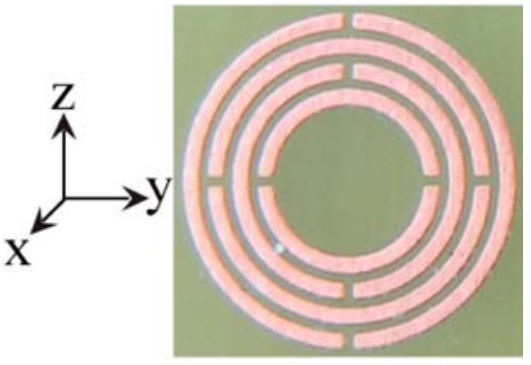

b)

Fig. 1 (online colour at: www.pss-b.com) a) Schematics of the labyrinth structure. $r 1=1.35 \mathrm{~mm}$, $r 2=1.8 \mathrm{~mm}, r 3=2.25 \mathrm{~mm}, r 4=2.7 \mathrm{~mm}, g=0.15 \mathrm{~mm}, w=0.3 \mathrm{~mm}$, and $d=0.15 \mathrm{~mm} . \mathrm{b})$ Unit cell of the actual, fabricated structure and the coordinate system that we used throughout the paper. The lattice constant is $8.8 \mathrm{~mm}$. 


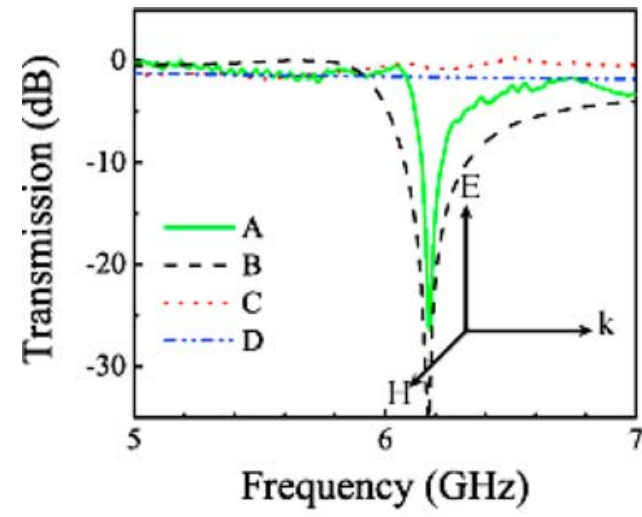

(a)

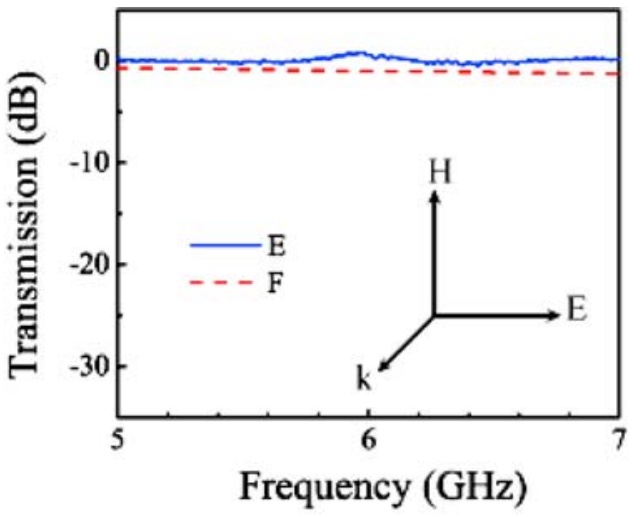

(b)

Fig. 2 (online colour at: www.pss-b.com) a) Measured transmission through a single labyrinth structure (A), calculated transmission through a single labyrinth structure (B), measured transmission through a single closed labyrinth structure (C), calculated transmission through a single closed labyrinth structure (D). b) Measured (E) and calculated (F) transmission through a single labyrinth structure.

labyrinth structure are shown in Fig. 2(b) for the incidence configuration when the magnetic resonance of an SRR structure can be excited by electric coupling. The transmission spectrum does not exhibit any resonant feature for this incidence configuration. Hence, these results show that the magnetic resonance of the labyrinth structure cannot be excited by electric coupling.

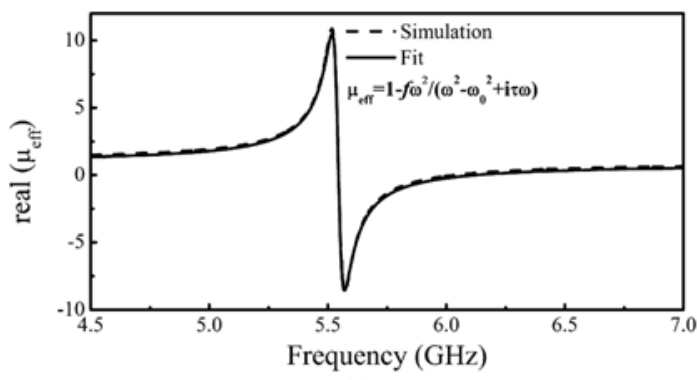

(a)

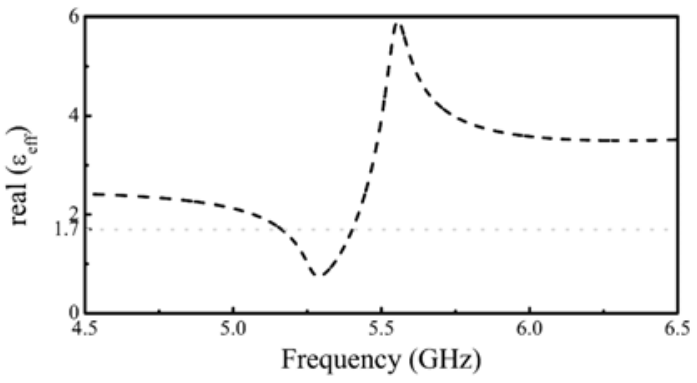

(c)

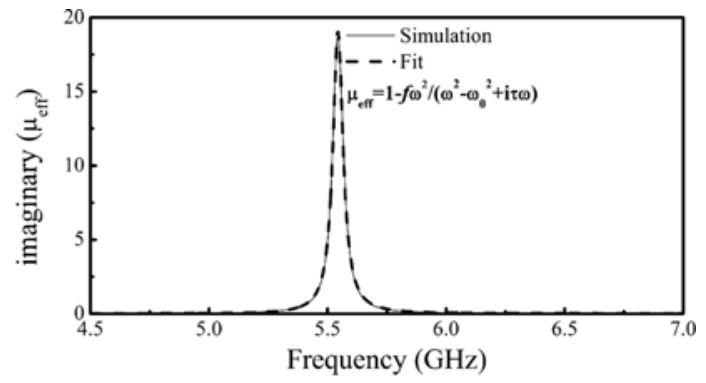

(b)

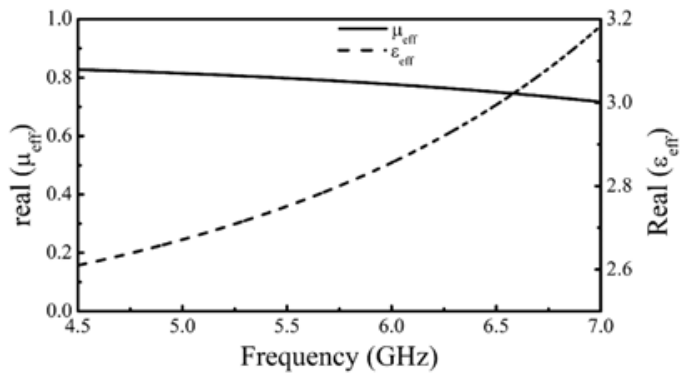

(d)

Fig. 3 a) Real part of the effective permeability for a single layer of the labyrinth structure, b) imaginary part of the effective permeability for a single layer of the labyrinth structure. c) Real part of the effective permittivity for a single layer of the labyrinth structure. d) Real part of the effective permittivity and permeability for a single layer of the closed labyrinth structure. 
We calculated the effective permittivities and permeabilities of the labyrinth based medium by use of a retrieval procedure $[27,28]$. The labyrinth medium consists of a periodic arrangement of single labyrinth structures. There was one layer of labyrinth structure along the direction of the propagation in our calculations. The orientation of the labyrinth structures was as shown Fig. 2(a). We employed periodic boundary conditions along directions other than the propagation direction. The dielectric constant of the PCB board was taken as 3.85. The PCB board had a thickness of $1.56 \mathrm{~mm}$. The effective permittivities and permeabilities were derived from the transmission and reflection coefficients. The retrieval results for a single labyrinth structure are plotted in Fig. 3(a)-(c). First of all, the real part of the effective permeability attained negative values above a certain frequency. The real and imaginary parts of the permeability fit quite well to the relation $\mu_{\mathrm{eff}}=1-f \omega^{2} /\left(\omega^{2}-\omega_{0}^{2}+i \tau \omega\right)$ [2]. We used the following fitting parameters for the real and imaginary parts of the effective permeability: $f=0.1806$ and $\tau=0.0526$. It is also interesting to observe that the imaginary part of the permeability attains quite high values in the close vicinity of the resonance frequency. This result indicates that the labyrinth structure shows a quite strong resonant behavior at the resonance frequency. The results for the permittivity are shown in Fig. 3(c). Notice that the permittivity is significantly increased just above the resonance frequency when compared to the effective permittivity of the host medium. The effective permittivity of the host medium was around 1.7 for our structure. This value was calculated by use of the relation $\varepsilon_{\text {eff }}=f \varepsilon_{\mathrm{i}}+(1-f) \varepsilon_{\mathrm{e}}$. At $5.55 \mathrm{GHz}$, the permittivity of the labyrinth structure attained a value, 5.92, which is 3.5 times larger than the effective permittivity of the host medium. Hence, one should consider the dielectric response of the labyrinth structure around the resonance frequency when designing LHM mediums. For comparison, we also plotted the retrieval results for the closed labyrinth medium in Fig. 3(d). The effective permeability of the closed labyrinth structure is less than one, but still positive in the frequency range that we considered. This result coincides well with the expectations, as the closed structure should be diamagnetic.

a)

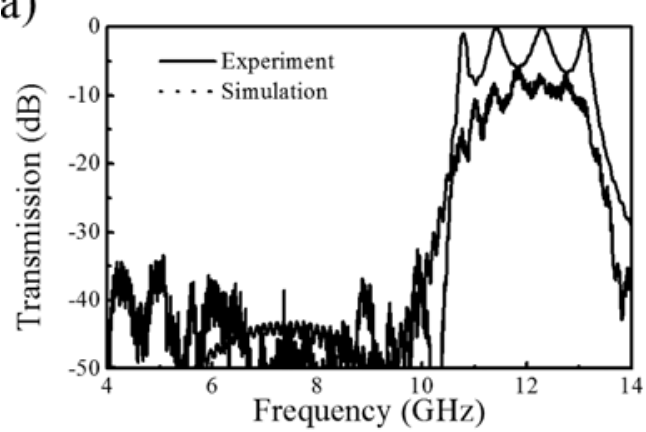

b)

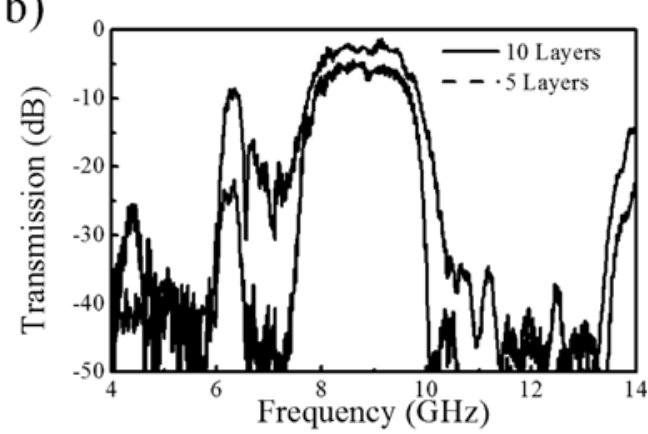

c)

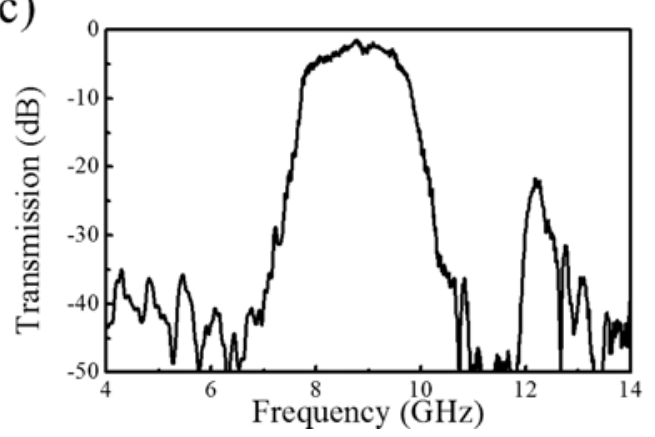

Fig. 4 a) Transmission spectrum of electromagnetic waves through the wire medium. b) Measured transmission spectrum of electromagnetic waves through the CMM medium. c) Measured transmission spectrum of electromagnetic wave through the closed CMM medium. 
The left-handed composite metamaterial (CMM) structure that we used in our study was composed of a one-dimensional periodic arrangement of labyrinth structures and wire structures. The wires were printed on the back surface of the PCBs, and labyrinth structures were fabricated on the front surface of the PCBs. The transmission spectrum of the wire only medium is shown in Fig. 4(a). The wire medium has a plasma frequency of approximately $10.45 \mathrm{GHz}$. For the CMM structure, the wires and labyrinth structures were aligned such that the axes of the wires were parallel to the splits on the labyrinth structure. There were 20 layers of CMM unit cells along the $z$-axis and 25 layers of CMM unit cells along the $x$-axis. The transmission spectrum for 5 and 10 layers of CMM unit cells along the propagation direction is shown in Fig. 4(b). Figure 4(b) shows that the transmission spectrum of the CMM medium exhibits a transmission band between $5.9 \mathrm{GHz}$ and $6.55 \mathrm{GHz}$. Note that the magnetic resonance of the single labyrinth structure was observed at $6.2 \mathrm{GHz}$. We measured the transmission spectrum of the closed CMM medium in order to check whether the transmission band observed between $5.9 \mathrm{GHz}$ and $6.55 \mathrm{GHz}$ is left-handed [29, 30]. The closed CMM medium consists of a periodic arrangement of closed labyrinth structures and wires strips. The lattice parameters were kept the same as the CMM medium. The transmission spectrum of the closed CMM medium is shown in Fig. 4(c). First of all, the transmission spectrum of the closed CMM medium did not exhibit a transmission band between $5.9 \mathrm{GHz}$ and $6.55 \mathrm{GHz}$. These results, therefore, show that the transmission band of the CMM medium is left-handed.

\section{Sub-wavelength focusing of electromagnetic waves by a two dimensional labyrinth based left-handed slab}

The two dimensional structure that we considered in this study consists of a periodic arrangement of wires and labyrinth structures in a two dimensional square lattice. The lattice constant was $8 \mathrm{~mm}$ along both of the lattice vectors of the square lattice. The unit cell of the two dimensional structure is shown in Fig. 5(a). The wire strips were printed on the back of the standard FR4 substrates and the labyrinths were printed on the front faces. The width of the wire stripes was $2.5 \mathrm{~mm}$. There were 68 layers along the $x$ direction and 5 layers along the y direction. The width of the structure was around one wavelength. The height of the structure was 20 layers long. The transmission spectrum of the structure is shown in Fig. 5(b). There is a left-handed transmission band between $6 \mathrm{GHz}$ and $6.4 \mathrm{GHz}$.

Pendry predicted that a slab of $\varepsilon=-1, \mu=-1$ may recover the evanescent components of the field emitted from a source [6]. In addition, due to negative refraction, such a medium focuses the propagating components of the source field. As a result, it may be possible to focus all of the Fourier components of the field emitted from a source. Pendry coined the term superlens for structures that have these proper-

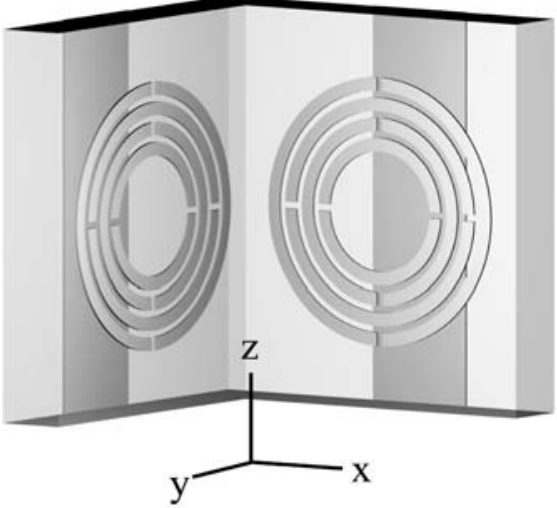

(a)

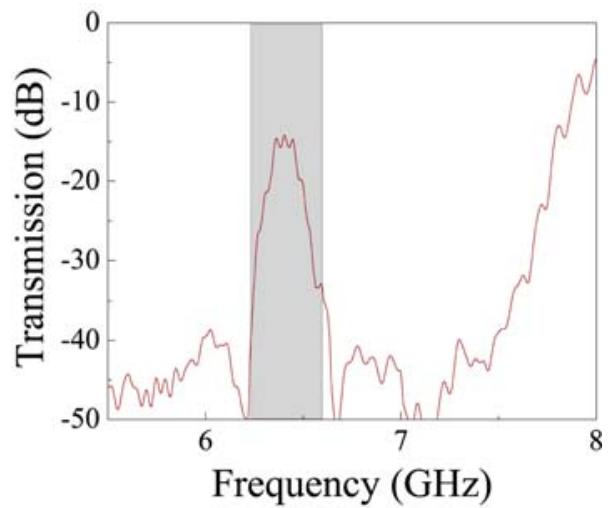

(b)

Fig. 5 (online colour at: www.pss-b.com) a) Unit cell of the two-dimensional labyrinth based lefthanded metamaterial. b) Transmission spectrum of two dimensional left-handed metamaterial medium. 


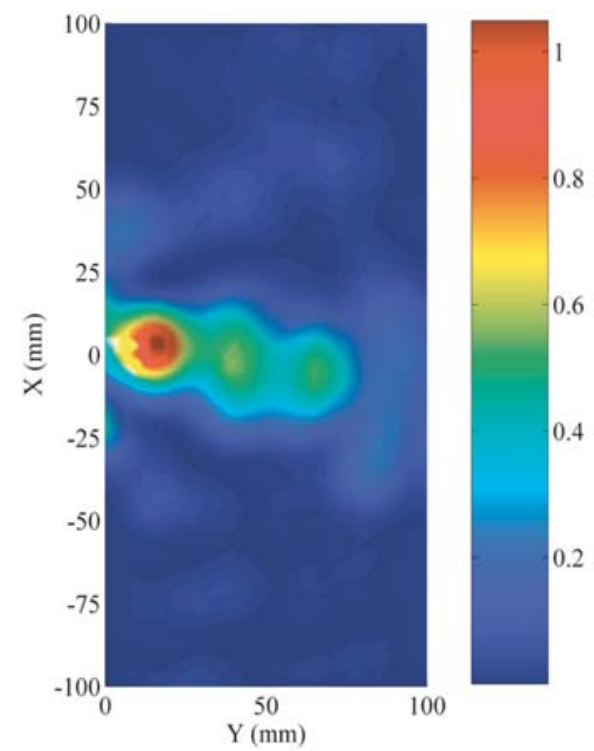

(a)

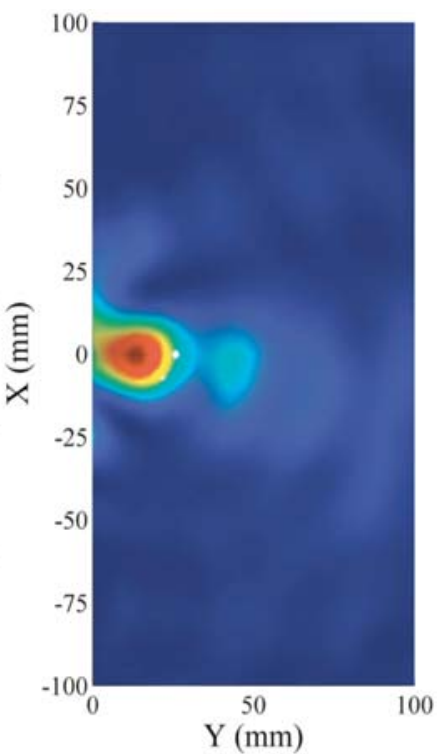

(b)

Fig. 6 (online colour at: www.pss-b.com) Measured electric field intensities on the output side of the metamaterial when the source was $2 \mathrm{~cm}$ away (a) and $1 \mathrm{~cm}$ away (b) from the input surface of the metamaterial.

ties. We checked the possibility of sub-wavelength focusing by use of the two dimensional labyrinth based left-handed medium.

We used monopole antennas as the source and receiver in our focusing studies. We placed the source antenna in front of the surface of the labyrinth based metamaterial. The axis of the source and receiver antennas were arranged parallel to the $z$-axis. We measured the electric field intensities over an area of $200 \times 100 \mathrm{~mm}^{2}$ on the output side of the left-handed metamaterial in steps of $2.5 \mathrm{~mm}$. The details of the experimental setup can be found elsewhere [7]. The measurements were carried out for two different source distances to the input surface, $10 \mathrm{~mm}$ and $20 \mathrm{~mm}$. The measurement results for source distances of $20 \mathrm{~mm}$ and $10 \mathrm{~mm}$ are shown in Fig. 6(a) and (b) for $6.3 \mathrm{GHz}$, respectively. The images of the source fields are clearly observed in Fig. 6(a) and (b). The maximum field intensity along the $y$-axes was observed at $10 \mathrm{~mm}$ when the source was $20 \mathrm{~mm}$ away from the input surface, in which it was $17.5 \mathrm{~mm}$ when the source was $10 \mathrm{~mm}$ away. The half widths of the intensity profiles along the $y$-axis for both cases were $19 \mathrm{~mm}$. On the other hand, the half width of the intensity profile on the output side of the metamaterial along the $x$-axes was $12 \mathrm{~mm}, \approx \lambda / 4$, when the source distance was $10 \mathrm{~mm}$. The measured half width of the intensity profile in free space, without the metamaterial in between, was $40 \mathrm{~mm}, \approx \lambda$. Without the metamaterial, even at such a close distance, the source field could not be resolved with a resolution below the wavelength. However, it was possible to the resolve the source field with a resolution below the wavelength when the metamaterial was inserted in between the source and receiver antennas.

\section{Transmission, refraction, and focusing properties of a three dimensional structure}

The three dimensional metamaterial that we used in this present study is shown in Fig. 7(a) While the structure is an extension of the two dimensional labyrinth based metamaterial to three dimensions, the fabrication of the structure is itself quite complicated. Particular attention was paid during the design 


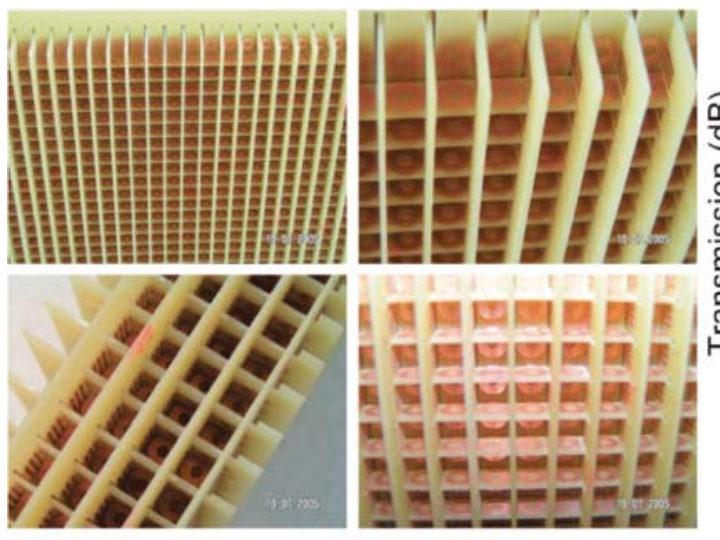

(a)

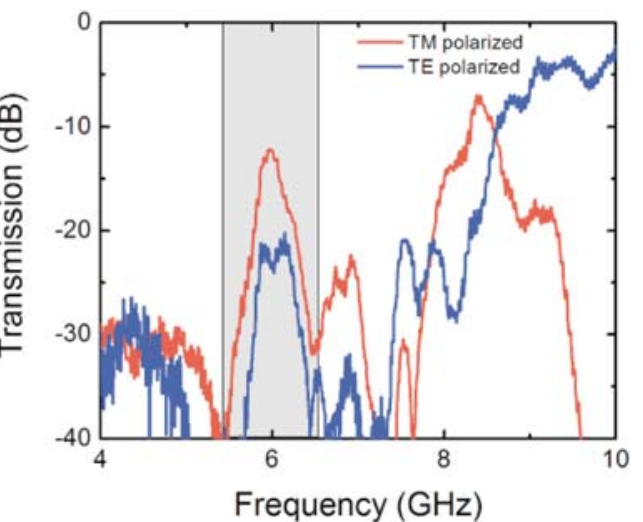

(b)

Fig. 7 (online colour at: www.pss-b.com) a) Photographs of the three dimensional structure. b) Measured transmission spectrum of TE and TM polarized waves through the labyrinth based three dimensional left-handed structure.

process in order to avoid intersecting wires. Such an intersection would evidently yield to a cut-wire medium. If not avoided, the cut-wire medium would cause us to loose the plasma-like behavior of a continuous wire medium. The results of the transmission measurements for TE and TM polarized electromagnetic waves, shown in Fig. 7(b), show the evidence of a left-handed transmission band between 5.7-6.35 GHz. TE and TM is used to denote two perpendicular polarizations each of which is parallel to different wire orientations in the labyrinth-based metamaterial. The transmission of TE waves is weak when compared to TM polarized waves. This difference in the transmission properties of TE-TM polarized waves indicates the presence of strong anisotropy. Such anisotropy is expected as the unit cell of the structure lacks the electrical symmetries in certain directions. Nonetheless, there is a left-handed transmission band for both TE and TM polarized waves that overlap in the frequency range.

In order to study the refraction properties of the $3 \mathrm{D}$ structure at the frequencies corresponding to the left-handed transmission band, we used electromagnetic waves incident on the structure at various angles. The setup that we used consists of transmitting horn antennas and receiving monopole antennas. The horn antenna was tilted such that the angles of incidence with respect to the normal vector of the

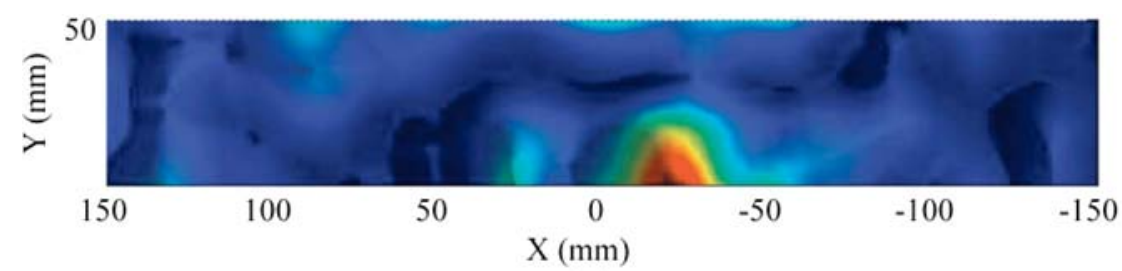

(a)

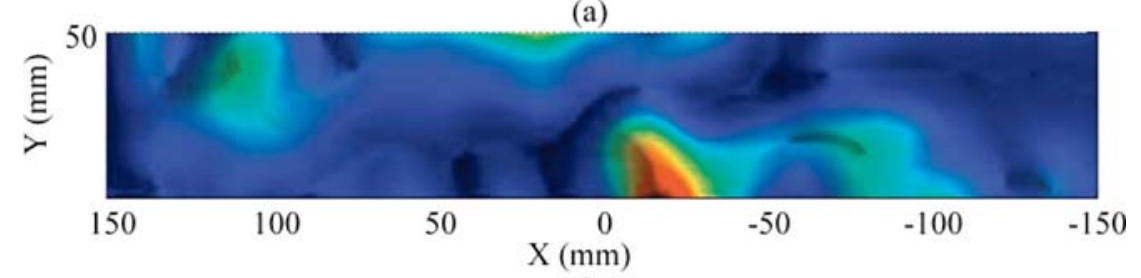

(b)

Fig. 8 (online colour at: www.pss-b.com) a) Measured electric field intensities for the incidence angle of 30 degrees. b) Measured electric field intensities for the incidence angle of 15 degrees. 

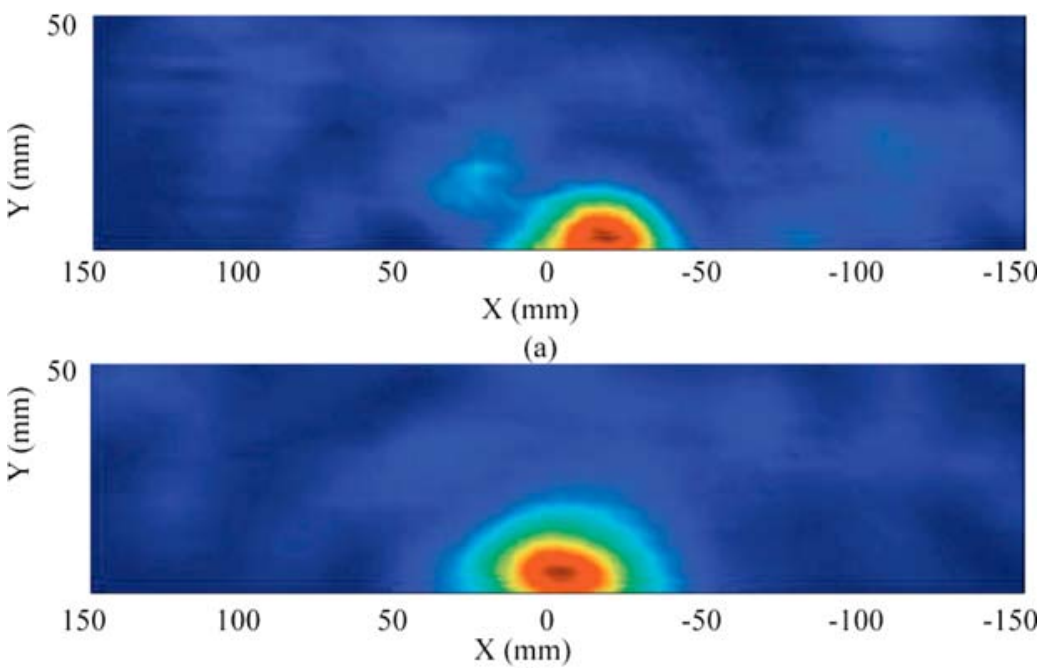

(b)

Fig. 9 (online colour at: www.pss-b.com) a) Measured electric field intensities for a source distance of $15 \mathrm{~mm}$. b) Measured electric field intensities for a source distance of $5 \mathrm{~mm}$.

structure were 15 and 30 degrees. The transmitted waves on the other side of the surface were measured by using the monopole antennas over a rectangular mesh. The details of the experimental setup can be found elsewhere [7]. The results of our measurements are shown in Fig. 8(a) and (b) for incidence angles of 15 and 30 degrees, respectively. The output surface was parallel to the $x$-axis. In addition, the waves were incident on the negative side with respect to the surface normal. Electromagnetic waves leave the output surface from the side of the surface normal that they were incident on. Hence, electromagnetic waves are negatively refracted by the $3 \mathrm{D}$ labyrinth based metamaterial. The index of refraction can be calculated by using the Snell's law and it is found to be around -1.02 at $6.2 \mathrm{GHz}$.

Since our 3D structure exhibited negative refraction, we next checked the focusing properties of the structure. We placed a monopole antenna in front of the input surface of the structure and measured the transmitted signal on the output side over a rectangular mesh by using a receiving monopole antenna. The results are shown in Fig. 9(a) and (b) for source distances of $15 \mathrm{~mm}$ and $5 \mathrm{~mm}$ to the input surface, respectively. First of all, one can clearly observe the images of the source field at the output side. In addition, the image of the source appears at a closer distance to the output surface for the source distance of $15 \mathrm{~mm}$ when compared to the source distance of $5 \mathrm{~mm}$. This result is expected for a metamaterial medium with simultaneously negative permittivity and permeability [6]. The half-width of the image is $24.5, \lambda / 2, \mathrm{~mm}$ at $6.1 \mathrm{GHz}$.

In conclusion, we studied the properties of one, two, and three dimensional labyrinth based lefthanded metamaterials. Our studies revealed that the labyrinth based medium overcomes the bianisotropy issue inherent to the split ring resonator based mediums. We were able to achieve sub-wavelength focusing by use of a two dimensional labyrinth based left-handed medium. Moreover, negative refraction of electromagnetic waves was demonstrated by the use of a 3D labyrinth based structure. In addition, we demonstrated the focusing of electromagnetic waves by use of a three dimensional flat lens. To our knowledge, this is the first experimental demonstration of a three dimensional left-handed medium that is based on resonant structures.

Acknowledgements This work is supported by the European Union under the projects EU-DALHM, EU-NOE-METAMORPHOSE, EU-NOE-PHOREMOST, and TUBITAK under Projects Nos. 104E090, 105E066, 105A005. One of the authors (Ekmel Özbay) also acknowledges partial support from the Turkish Academy of Sciences. 


\section{References}

[1] V. G. Veselago, Sov. Phys. Usp. 10, 509 (1968).

[2] D. R. Smith, Willie J. Padilla, D. C. Vier, S. C. Nemat-Nasser, and S. Schultz, Phys. Rev. Lett. 84, 4184 (2000).

[3] C. G. Parazzoli, R. B. Greegor, K. Li, B. E. C. Koltenbah, and M. Tanielian, Phys. Rev. Lett. 90, 107401 (2003).

[4] A. A. Houck, J. B. Brock, and I. L. Chuang, Phys. Rev. Lett. 90, 137401 (2003).

[5] A. M. Belyantsev and A. B. Kozyrev, Tech. Phys. 47, 1477 (2002).

[6] J. B. Pendry, Phys. Rev. Lett. 85, 3966 (2000).

[7] K. Aydin, I. Bulu, and E. Özbay, Opt. Express 13, 7645-7652 (2005).

[8] I. Bulu, H. Caglayan, and E. Özbay, Opt. Lett. 31, 814-816 (2006).

[9] A. Grbic and G. V. Eleftheriades, Appl. Phys. Lett. 82, 1815 (2003).

[10] I. Bulu, H. Caglayan, and E. Özbay, Opt. Express 13, 10238 (2005).

[11] J. B. Pendry, A. J. Holden, D. J. Robins, and W. J. Stewart, IEEE Trans. Microw. Theory Tech. 47, 2075 (1999).

[12] P. Markos and C. M. Soukoulis, Phys. Rev. B 65, 033401 (2001).

[13] Mehmet Bayindir, K. Aydin, E. Özbay, P. Marko, and C. M. Soukoulis, Appl. Phys. Lett. 81, 120 (2002).

[14] Ph. Gay-Balmaz and O. J. F. Martin, J. Appl. Phys. 92, 2929 (2002).

[15] R. Marques, J. Martel, F. Mesa, and F. Medina, Phys. Rev. Lett. 89, 183901 (2002).

[16] C. R. Simovski and B. Sauviac, Phys. Rev. E 70, 046607 (2004).

[17] A. B. Movchan and S. Guenneau, Phys. Rev. B 70, 125116 (2004).

[18] Yi-Jang Hsu, Yen-Chun Huang, Jiann-Shing Lih, and Jyh-Long Chern, J. Appl. Phys. 96, 1979 (2004).

[19] M. Shamonin, E. Shamonina, V. Kalinin, and L. Solymar, J. Appl. Phys. 95, 3778 (2004).

[20] Yen-Chun Huang, Yi-Jang Hsu, Jiann-Shing Lih, and Jyh-Long Chern, Jpn. J. Appl. Phys., Part 2 43, L190 (2004).

[21] B. Sauviac, C. R. Simovski, and S. A. Tretyakov, Electromagnetics 24, 317 (2004).

[22] Philippe Gay-Balmaz and Olivier J. F. Martin, Appl. Phys. Lett. 81, 939 (2002).

[23] Xudong Chen, Bae-Ian Wu, Jin Au Kong, and T. M. Grzegorczyk, Phys. Rev. E 71, 046610 (2005).

[24] R. Marques, F. Mesa, J. Martel, and F. Medina, IEEE Trans. Antennas Propag. 51, 2572 (2003).

[25] R. Marques, F. Medina, and R. Rafii-El-Idrissi, Phys. Rev. B 65, 144440 (2002).

[26] N. Katsarakis, T. Koschny, M. Kafesaki, E. N. Economou, and C. M. Soukoulis, Appl. Phys. Lett. 84, 2943 (2004).

[27] Xudong Chen, T. M. Grzegorczyk, Bae-Ian Wu, J. Pacheco, Jr., and Jin Au Kong, Phys. Rev. E 70, 016608 (2004).

[28] Xudong Chen, Bae-Ian Wu, Jin Au Kong, and T. M. Grzegorczyk, Phys. Rev. E 71, 046610 (2005).

[29] T. Koschny, M. Kafesaki, E. N. Economou, and C. M. Soukoulis, Phys. Rev. Lett. 93, 107402 (2004).

[30] K. Aydin, K. Guven, M. Kafesaki, Lei Zhang, C. M. Soukoulis, and E. Özbay, Opt. Lett. 29, 2623 (2004). 